\title{
Publicidad y promoción de medicamentos: regulaciones y grado de acatamiento en cinco países de América Latina
}

\author{
Claudia Vacca, ${ }^{1}$ Claudia Vargas, ${ }^{2}$ Martín Cañás ${ }^{3}$ y Ludovic Reveiz ${ }^{4}$
}

Forma de citar

Vacca C, Vargas C, Cañás M, Reveiz L. Publicidad y promoción de medicamentos: regulaciones y grado de acatamiento en cinco países de América Latina. Rev Panam Salud Publica. 2011;29(2):76-83.

RESUMEN Objetivo. Analizar las distintas regulaciones sobre promoción de fármacos y su grado de acatamiento reflejado en piezas publicitarias expuestas al público en Argentina, Colombia, Ecuador, Nicaragua y Perú.

Métodos. Se recogieron 683 piezas promocionales expuestas en establecimientos de salud, farmacias y en la vía pública, de las cuales 132 piezas seleccionadas al azar fueron objeto de análisis. Se examinaron las regulaciones sobre publicidad farmacéutica -incluidas sus coincidencias con los criterios éticos de la Organización Mundial de la Salud (OMS) — tomadas de los sitios web oficiales y mediante entrevistas con los responsables de los organismos regulatorios y ministerios de salud de los cinco países del estudio. Se evaluaron los contenidos de los materiales de la muestra para determinar su grado de acatamiento respecto a las regulaciones nacionales y las recomendaciones sobre promoción de medicamentos de la OMS.

Resultados. Los países cuentan con regulaciones que incorporan los criterios éticos de la OMS. Más de $80 \%$ de las piezas analizadas incluían las indicaciones del fármaco y más de 70\% omitían información sobre efectos adversos. Cincuenta por ciento de los anuncios de medicamentos de venta libre (MVL) expuestos en farmacias incluían indicaciones no aprobadas por la autoridad sanitaria correspondiente. En los anuncios expuestos en farmacias, no se hallaron diferencias significativas entre los riesgos de la información inadecuada con relación a su condición de venta (MVL o medicamentos de venta con prescripción médica). El riesgo relativo de ausencia de información sobre posología fue de 2,08 (intervalo de confianza de 95\% 1,32-3,39) en las piezas distribuidas en farmacias, comparadas con las expuestas en establecimientos de salud. Conclusiones. Si bien en general los cinco países del estudio incorporan en sus regulaciones sobre promoción y publicidad de medicamentos las recomendaciones de la OMS, con frecuencia dichas ordenanzas no se reflejan en los contenidos de las piezas promocionales.

Palabras clave Publicidad de medicamentos; ética farmacéutica; control de la publicidad de productos; medicamentos sin prescripción; medicamentos bajo prescripción; Argentina; Colombia; Ecuador; Nicaragua; Perú; América Latina.

1 Universidad Nacional de Colombia; Red para el Uso Adecuado de Medicamentos (RAM), Bogotá, Colombia. La correspondencia se debe dirigir a Claudia Vacca, cpvacag@bt.unal.edu.co

2 Fundación Ifarma; Acción Internacional por la Salud (AIS). Bogotá, Colombia.

3 Fundación Femeba; Grupo Argentino para el Uso Racional de Medicamentos (GAPURMED).

4 Cuando se realizó el estudio: Fundación Sanitas Internacional, Instituto de Investigaciones. Bogotá, Colombia. Actualmente: Organización Panameri-
En 1988 la Organización Mundial de la Salud (OMS) publicó los "Criterios éticos para la promoción de medicamentos" con el propósito de garantizar que las actividades de marketing sean compatibles con la búsqueda de la verdad y la rectitud que

cana de la Salud, Washington D.C., Estados Unidos de América. deben guardar la promoción y la publicidad farmacológicas (1). Más adelante emitió otros pronunciamientos dirigidos a fortalecer la normativa sobre la promoción de medicamentos - y su cumplimiento- así como un llamado de atención acerca de la promoción, publicidad y venta transfronteriza de productos médicos en Internet, 
donde ya se habían detectado prácticas éticamente conflictivas (2-5).

La transparencia de la promoción farmacéutica, entendida como veracidad y responsabilidad en la divulgación, busca que los consumidores estén bien informados tanto sobre los beneficios como sobre los posibles riesgos relacionados con los fármacos que se les están prescribiendo $(6,7)$. Tal consigna parte del supuesto de que los pacientes en general no tienen conocimientos suficientes como para discernir la veracidad de un mensaje publicitario sobre medicamentos (8).

$\mathrm{Si}$ bien gran parte de las regulaciones de la industria farmacéutica establecen que la promoción y publicidad en medios de comunicación masiva deben limitarse a los medicamentos de venta libre (MVL) y las prohíben explícitamente para los de venta con prescripción médica (MVP), las ambigüedades presentes en las definiciones de promoción, publicidad y lo que constituye información médica, pueden y suelen dar paso a actividades de difusión comercial masiva disimulada (9). En nada ayuda tampoco la creciente presión de la industria farmacéutica por autorregularse mediante la búsqueda de códigos de ética propios, promovidos por la Federación Internacional de Asociaciones de Fabricantes Farmacéuticos, que pueden distraer o reducir el papel fiscalizador de los organismos de la salud (10).

En los últimos años, investigaciones realizadas en Estados Unidos, Canadá, Australia, Nueva Zelandia, España y Brasil revelan hallazgos preocupantes relacionados con la publicidad de fármacos en los medios de comunicación masiva (11-16). Un estudio publicado recientemente señala que la propaganda farmacológica directa al consumidor genera un aumento de las ventas de los productos anunciados (17). La información difundida por estos medios, entonces, puede incidir no solo en la idoneidad de la práctica médica sino además en la salud y el bienestar del paciente, así como en los costos de la atención, los cuales son generalmente asumidos por el gobierno o por el mismo paciente, sea mediante su cobertura médica o con dinero de su propio bolsillo $(18,19)$.

Con estos antecedentes, el presente trabajo se propuso analizar las distintas regulaciones sobre promoción de fármacos y su grado de acatamiento reflejado en piezas publicitarias expuestas al público en Argentina, Colombia, Ecuador, Nicaragua y Perú.

\section{MATERIALES Y MÉTODOS}

Se realizó un estudio descriptivo de corte transversal para examinar y evaluar las regulaciones sobre promoción farmacéutica de cinco países - Argentina, Colombia, Ecuador, Nicaragua y Perú- de América Latina, así como los contenidos de las piezas promocionales disponibles al público y al personal de salud, con base en los lineamientos de los "Criterios éticos para la promoción de medicamentos" de la OMS (1).

Con objeto de obtener información sobre regulaciones relacionadas con la promoción de medicamentos, se entrevistó $^{5}$ a los responsables de fiscalizar las actividades publicitarias de fármacos en los ministerios y otros organismos de salud de los cinco países estudiados, quienes - personalmente o vía correo electrónico- respondieron un cuestionario estructurado con preguntas sobre normas y regulaciones que incluyeron componentes tales como medios de difusión permitidos, requisitos de la información al público, restricciones a la información, patrocinio de eventos y otras actividades promocionales, así como prácticas de promoción prohibidas y sus sanciones pertinentes. Estos datos fueron confrontados entre noviembre y diciembre de 2007 con una revisión exhaustiva de las regulaciones nacionales sobre promoción de fármacos (leyes, decretos, resoluciones, estándares, directivas y otras normativas) a través de los sitios web de las entidades sanitarias participantes y una consulta de actualización en septiembre de 2009.

Tras ser evaluados y consolidados, los contenidos regulatorios fueron remitidos al coordinador del estudio, quien los incorporó en una matriz comparativa por país que luego fue valorada por investigadores de Argentina y Colombia de manera simultánea, no cegada.

El análisis se realizó con un esquema predeterminado, identificando brechas entre los criterios éticos de la OMS, por un lado, y el contenido regulatorio por otro, considerándose los grados de restricción (presencia detallada y ampliada de los criterios de la OMS) y de flexibilidad (ausencia o menor detalle de los criterios de la OMS) entre ambos.

\footnotetext{
El cuestionario fue administrado por los autores y por miembros del Grupo de Análisis y Vigilancia de la Promoción Farmacéutica (GAViP) de la red de Acción Internacional por la Salud (AIS).
}

Adicionalmente, entre enero y septiembre de 2008 se recogieron piezas publicitarias de MVP y MVL difundidas por diferentes medios y dirigidas tanto a los profesionales de la salud como al público en general de los cinco países estudiados. Se definieron como "piezas publicitarias" todos los materiales de promoción impresos -afiches, carteles, encartes, folletos, gigantografías, paneles, plegables y volantes- destinados a profesionales de la salud y a la población en general. No se incluyeron anuncios publicados en revistas o divulgados por internet, ni materiales de promoción audiovisual.

Las piezas promocionales se recolectaron en establecimientos de atención de salud públicos y privados — consultorios de hospitales públicos, clínicas privadas, consultorios particulares, farmacias y boticas - seleccionados por conveniencia, prefiriendo los sitios de más fácil acceso para los investigadores. Se actuó bajo la premisa de que usualmente las campañas de marketing de medicamentos no son selectivas en cuanto a lugares específicos, considerándose en consecuencia que, más que el lugar, lo relevante era el material publicitario disponible.

En cada uno de los países la recolección de piezas de publicidad se llevó a cabo en el lapso de un mes, período durante el cual se visitaron como mínimo cinco instituciones públicas y privadas, y cinco farmacias o boticas. La búsqueda se extendió asimismo a los alrededores de los establecimientos de salud, donde se identificaron y fotografiaron paneles, afiches y otros materiales de publicidad farmacéutica en calles y plazas y en vehículos de transporte público.

Se estimó que al menos 30\% de las piezas omitiría información importante acerca de eventos adversos o incluiría indicaciones no aprobadas por la autoridad sanitaria correspondiente. Con un nivel de confianza de $95 \%$ y un peor resultado de $23 \%$, se calculó una muestra de un mínimo de 100 piezas promocionales para analizar. Del total del material recolectado se retiraron las piezas repetidas y se realizó un inventario de los materiales según su formato. En cada país se asignó un número a cada una de las piezas. Posteriormente, de una bolsa de papeletas que contenía los números de cada una de las piezas recolectadas, se seleccionaron 15 materiales publicitarios provenientes de los establecimientos de atención de salud públicos y privados y 15 provenientes de farmacias y boticas de 
cada país, desconociéndose las proporciones de MVP y MVL correspondientes.

La información fue recogida por un evaluador debidamente entrenado y se dispuso además de un folleto instructivo acerca de cómo recoger y procesar los datos en cada país. Con pocas excepciones (p. ej. la condición de venta del producto [MVP o MVL]), los datos se extrajeron únicamente del material publicitario objeto de evaluación. Todos los materiales fueron remitidos para su evaluación a Colombia, donde se creó una base única de datos que permitiría verificar los contenidos de cada país, disminuir inconsistencias y evitar duplicidad en las piezas analizadas.

Las piezas promocionales se analizaron según una ficha de evaluación previamente diseñada. Las variables generales incluían el país de origen, el sitio de la recolección (establecimientos públicos o privados, farmacias, boticas y vía pública) y el tipo de pieza promocional (afiches, carteles, encartes, folletos, gigantografías y otros). Las variables asociadas a la información analizada de cada pieza publicitaria incluían la marca, el principio activo, la condición de venta del producto (cuando el dato no aparecía en el anuncio se obtuvo de la entidad de salud), el fabricante, la empresa que lo vendía, las indicaciones para las cuales se promovía el producto, las referencias bibliográficas citadas y la información del producto (reacciones adversas, precauciones/advertencias, contraindicaciones, posología y forma farmacéutica).

La evaluación de los materiales se realizó tomando como estándares datos técnicos del Formulario Nacional Británico y el Centro para la Evaluación e Investigación de Medicamentos -Administración de Alimentos y Medicamentos de los Estados Unidos- así como, cuando estaban disponibles, los registros sanitarios de los productos (ficha nacional de referencia) que permitían verificar las indicaciones aprobadas (20-22). Para el análisis estadístico se utilizó el programa estadístico SPSS $11.0^{\circledR}$.

Los resultados de las variables analizadas se presentan en porcentajes en razón de que no pueden ser consideradas categóricas. Se estimaron las medidas de asociación y el riesgo relativo (RR) con sus intervalos de confianza, tomando como referencia para comparar el tipo de información contenida en las piezas promocionales según el lugar de recolección de la información (farmacias o instituciones de salud) y la condición de venta del medicamento (MVP/MVL).

\section{RESULTADOS}

\section{Regulación de la promoción farmacéutica}

Algunas de las regulaciones consultadas distinguen entre publicidad y promoción ( $\mathrm{P}$ y $\mathrm{P}$ ) de medicamentos, en tanto que otras las consideran como una unidad. En Colombia, por ejemplo, se establece que "la publicidad es una actividad informativa y la promoción una actividad de orientación", mientras que en los otros cuatro países se hace referencia específica a la P y P como estrategia de inducción al consumo o a la adquisición de medicamentos.

En los cinco países del estudio, la P y P en medios masivos de comunicación se restringe a MVL y se prohíbe explícitamente para MVP (23-27). Y todos, en consonancia con los criterios establecidos por la OMS, disponen que la información del mensaje publicitario debe ser "veraz, clara y objetiva; fidedigna, exacta, verdadera, informativa y equilibrada; actualizada, educativa e inequívoca, susceptible de comparación y de buen gusto" (1).

Las regulaciones sobre P y P de MVL son más flexibles en Argentina, Colombia y Ecuador que en Nicaragua y Perú, mientras que para MVP todas las regulaciones analizadas parecen restringidas con respecto a las mismas variables en referencia a los criterios éticos de la OMS (cuadro 1).

CUADRO 1. Características de las regulaciones sobre promoción y publicidad de medicamentos, según sean de venta libre o requieran prescripción médica, en cinco países de América Latina

\begin{tabular}{|c|c|c|c|c|}
\hline \multirow[b]{2}{*}{ País } & \multirow[b]{2}{*}{$\begin{array}{l}\text { Medios de difusión } \\
\text { permitidos }\end{array}$} & \multicolumn{2}{|c|}{ Requisitos según el destinatario } & \multirow[b]{2}{*}{$\begin{array}{l}\text { Restricciones de } \\
\text { la información }\end{array}$} \\
\hline & & $\begin{array}{l}\text { Público } \\
\text { general }\end{array}$ & $\begin{array}{c}\text { Profesionales } \\
\text { de la salud }\end{array}$ & \\
\hline \multicolumn{5}{|c|}{ Medicamentos de venta libre } \\
\hline Argentina & Sí & Sí & NA & Sí \\
\hline Colombia & Sí & Sí restringida & NA & Sí restringida \\
\hline Ecuador & No & Sí laxa & NA & No \\
\hline Nicaragua & Sí & Sí restringida & NA & Sí restringida \\
\hline Perú & Sí & Sí restringida & NA & Sí restringida \\
\hline \multicolumn{5}{|c|}{ Medicamentos de venta con prescripción } \\
\hline Argentina & Sí laxa & Sí restringida & Sí restringida & No \\
\hline Colombia & Sí restringida & Sí laxa & Sí restringida & Sí restringida \\
\hline Ecuador & Sí restringida & No & No & Sí laxa \\
\hline Nicaragua & Sí restringida & Sí restringida & Sí restringida & Sí restringida \\
\hline Perú & Sí restringida & Sí laxa & Sí laxa & Sí restringida \\
\hline
\end{tabular}

Fuente: elaboración de los autores con base en las referencias 29 a 33.

Nota: Sí: el tópico es tratado de manera expresa en la regulación. No: el tópico no es tratado de manera expresa en la regulación. Restringida: el tópico aparece en forma más detallada que en los criterios éticos de la Organización Mundial de la Salud (OMS). Laxa: el tópico aparece en forma menos detallada que en los criterios éticos de la OMS. NA: datos no aplicables. 
CUADRO 2. Características de las 132 piezas promocionales de medicamentos analizadas en cinco países de América Latina

\begin{tabular}{|c|c|c|c|c|c|c|c|c|c|c|c|}
\hline \multirow[b]{3}{*}{ País } & \multirow[b]{3}{*}{$n$} & \multicolumn{10}{|c|}{ No. de piezas (\%) } \\
\hline & & \multicolumn{2}{|c|}{ Condición de venta } & \multicolumn{5}{|c|}{ Formato } & \multicolumn{3}{|c|}{ Lugar de recolección } \\
\hline & & Prescripción & Libre & Afiche & Folleto & Volante & Plegables & Otros & $\begin{array}{c}\text { Establecimiento } \\
\text { de salud }\end{array}$ & Farmacia & Vía pública \\
\hline Nicaragua & 30 & $\begin{array}{c}27 \\
(90)\end{array}$ & $\begin{array}{c}3 \\
(10)\end{array}$ & $\begin{array}{c}4 \\
(13,3)\end{array}$ & 0 & $\begin{array}{c}11 \\
(36,7)\end{array}$ & $\begin{array}{c}10 \\
(33,3)\end{array}$ & $\begin{array}{c}5 \\
(16,7)\end{array}$ & $\begin{array}{c}14 \\
(48,2)\end{array}$ & $\begin{array}{c}15 \\
(51,8)\end{array}$ & 0 \\
\hline Argentina & 29 & $\begin{array}{l}20 \\
(69)\end{array}$ & $\begin{array}{c}9 \\
(31)^{\mathrm{a}}\end{array}$ & 0 & $\begin{array}{c}12 \\
(41,4)\end{array}$ & $\begin{array}{c}10 \\
(34,5)\end{array}$ & 0 & $\begin{array}{c}7 \\
(24,1)\end{array}$ & $\begin{array}{c}15 \\
(50)\end{array}$ & $\begin{array}{c}15 \\
(50)\end{array}$ & 0 \\
\hline Perú & 30 & $\begin{array}{c}15 \\
(50)\end{array}$ & $\begin{array}{c}15 \\
(50)\end{array}$ & $\begin{array}{c}2 \\
(6,7)\end{array}$ & $\begin{array}{c}8 \\
(26,7)\end{array}$ & $\begin{array}{c}7 \\
(23,3)\end{array}$ & $\begin{array}{c}6 \\
(20)\end{array}$ & $\begin{array}{c}7 \\
(23,3)\end{array}$ & $\begin{array}{c}15 \\
(50)\end{array}$ & $\begin{array}{c}15 \\
(50)\end{array}$ & 0 \\
\hline Ecuador & 10 & $\begin{array}{c}7 \\
(70)\end{array}$ & $\begin{array}{c}3 \\
(30)\end{array}$ & $\begin{array}{c}2 \\
(20)\end{array}$ & $\begin{array}{c}2 \\
(20)\end{array}$ & 0 & $\begin{array}{c}6 \\
(60)\end{array}$ & 0 & $\begin{array}{c}7 \\
(70)\end{array}$ & $\begin{array}{c}3 \\
(30)\end{array}$ & 0 \\
\hline Total & 132 & $\begin{array}{c}92 \\
(69,7)\end{array}$ & $\begin{array}{c}40 \\
(30,3)\end{array}$ & $\begin{array}{c}15 \\
(11,4)\end{array}$ & $\begin{array}{c}43 \\
(32,6)\end{array}$ & $\begin{array}{c}28 \\
(21,2)\end{array}$ & $\begin{array}{c}22 \\
(16,7)\end{array}$ & $\begin{array}{c}24 \\
(18,2)\end{array}$ & $\begin{array}{c}64 \\
(48,5)\end{array}$ & $\begin{array}{c}67 \\
(50,8)\end{array}$ & $\begin{array}{c}1 \\
(0,8)\end{array}$ \\
\hline
\end{tabular}

Fuente: elaboración de los autores.

a No se especifica la condición de venta en una de las piezas analizadas.

CUADRO 3. Lugar de recolección de las 132 piezas promocionales y condición de venta del medicamento promocionado en cinco países de América Latina

\begin{tabular}{|c|c|c|c|c|c|c|c|}
\hline \multirow[b]{3}{*}{ País } & \multicolumn{7}{|c|}{ No. de piezas (\%) } \\
\hline & \multicolumn{3}{|c|}{ Venta con prescripción } & \multicolumn{4}{|c|}{ Venta libre } \\
\hline & $n$ & $\begin{array}{l}\text { Establecimientos } \\
\text { de salud }^{\mathrm{a}}\end{array}$ & Farmacias & $n$ & $\begin{array}{l}\text { Establecimientos } \\
\text { de salud }^{\mathrm{a}}\end{array}$ & Farmacias & $\begin{array}{l}\text { Vía } \\
\text { pública }\end{array}$ \\
\hline Nicaragua & 27 & $\begin{array}{c}15 \\
(55,6)\end{array}$ & $\begin{array}{c}12 \\
(44,4)\end{array}$ & 3 & 0 & $\begin{array}{c}3 \\
(100)\end{array}$ & 0 \\
\hline Argentina & 20 & $\begin{array}{l}13 \\
(65)\end{array}$ & $\begin{array}{c}7 \\
(35)\end{array}$ & 9 & $\begin{array}{c}1 \\
(11,1)\end{array}$ & $\begin{array}{c}8 \\
(88,9)\end{array}$ & 0 \\
\hline Colombia & 23 & $\begin{array}{c}12 \\
(52,2)\end{array}$ & $\begin{array}{c}11 \\
(47,8)\end{array}$ & 10 & $\begin{array}{c}1 \\
(10)\end{array}$ & $\begin{array}{c}8 \\
(80)\end{array}$ & $\begin{array}{c}1 \\
(10,0)\end{array}$ \\
\hline Perú & 15 & $\begin{array}{c}13 \\
(86,7)\end{array}$ & $\begin{array}{c}2 \\
(13,3)\end{array}$ & 15 & $\begin{array}{c}2 \\
(13,3)\end{array}$ & $\begin{array}{c}13 \\
(86,7)\end{array}$ & 0 \\
\hline Ecuador & 7 & $\begin{array}{c}5 \\
(71,4)\end{array}$ & $\begin{array}{c}2 \\
(28,6)\end{array}$ & 3 & $\begin{array}{c}2 \\
(66,7)\end{array}$ & $\begin{array}{c}1 \\
(33,3)\end{array}$ & 0 \\
\hline Total & 92 & $\begin{array}{l}58 \\
(63)\end{array}$ & $\begin{array}{l}34 \\
(37)\end{array}$ & 40 & $\begin{array}{c}6 \\
(15)\end{array}$ & $\begin{array}{c}33 \\
(82,5)\end{array}$ & $\begin{array}{c}1 \\
(2,5)\end{array}$ \\
\hline
\end{tabular}

Fuente: elaboración de los autores.

a En esta categoría se incluyen hospitales públicos, clínicas privadas y consultorios médicos.

establecimientos de salud, respectivamente (cuadro 4). La información más frecuentemente omitida en piezas promocionales sobre MVL expuestas en farmacias y en la vía pública fue la relacionada con la seguridad de los fármacos: reacciones adversas $(85,3 \%)$, contraindicaciones $(61,8 \%)$ y precaucionesadvertencias $(52,9 \%)$. La mención de indicaciones no autorizadas en materiales sobre MVL hallados en farmacias y en la vía pública alcanzó un promedio de $50 \%$ (17 de 34), presentando Ecuador el valor más alto (100\%) con una pieza analizada, seguido por Argentina (75\%) con una muestra mayor (cuadro 4).

Cuando se evaluó el riesgo de ausencia de información sobre indicaciones del medicamento en función del sitio donde se recogió el material de promoción, se encontró un RR de 10,24 (intervalo de confianza de 95\% [IC95\%] 1,29-81,46) en las farmacias en comparación con los establecimientos de salud. El riesgo de ausencia de información sobre posología en materiales publicitarios distribuidos en farmacias fue el doble del correspondiente a los distribuidos en establecimientos, RR 2,08 (IC95\% 1,32-3,29). No se encontraron diferencias significativas entre la información promocional contenida en las piezas promocionales de MVL y MVP sobre indicaciones, reacciones adversas, precauciones y advertencias, contraindicaciones, posología, formas farmacéuticas e indicaciones no aprobadas por la autoridad sanitaria correspondiente (cuadro 5).

\section{DISCUSIÓN}

\section{Regulación}

Los resultados del presente trabajo coinciden con los de otros estudios que observaron que, si bien en general las regulaciones de los países sobre publicidad de medicamentos toman como referencia los criterios éticos de la OMS, hay escasa información sobre vigilancia y no se contempla el papel de los consumidores ni de otras entidades en el monitoreo de las actividades promocionales $(9,28,29)$.

Asimismo, se detectó la ausencia de algunos criterios clave para la prevención de riesgos y protección de los consumidores, así como ambigüedades en el uso de algunos términos. Por ejemplo, en algunas regulaciones las definiciones de promoción, publicidad e información médica podrían facilitar la difusión masiva de promoción de forma disimulada. Esta ambigüedad ya ha sido señalada y también la recomendación de fortalecer el papel del Estado en el control de la $\mathrm{P}$ y $P(30)$.

\section{Piezas promocionales}

La información más frecuentemente omitida en piezas promocionales sobre MVP es la relacionada con la seguridad. Aun así, el hallazgo de mayor preocupación podría ser el elevado porcentaje de información relacionada con indica- 
CUADRO 4. Características del contenido de las 132 piezas promocionales de medicamentos seleccionadas y analizadas, según el lugar de recolección, en cinco países de América Latina

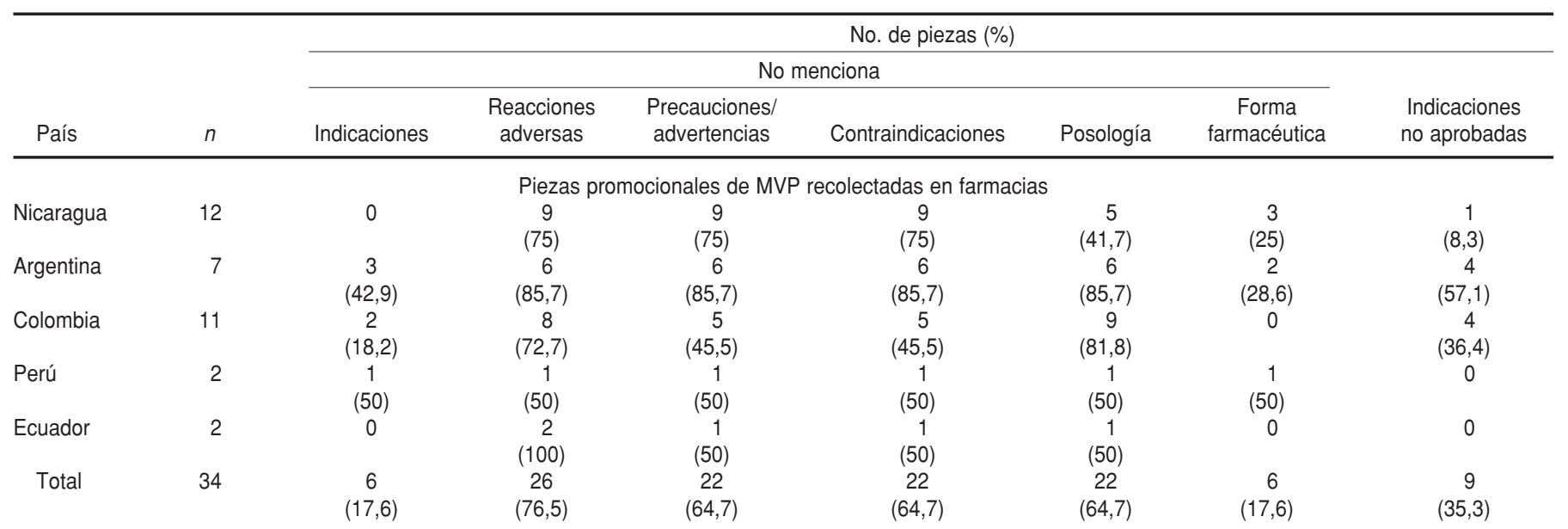

Piezas promocionales de MVP recolectadas en establecimientos de salud

\begin{tabular}{|c|c|c|c|c|c|c|c|c|}
\hline Nicaragua & 15 & 0 & $\begin{array}{c}10 \\
(66,7)\end{array}$ & $\begin{array}{c}8 \\
(53,3)\end{array}$ & $\begin{array}{c}8 \\
(53,3)\end{array}$ & $\begin{array}{c}4 \\
(26,7)\end{array}$ & 0 & $\begin{array}{c}3 \\
(20,0)\end{array}$ \\
\hline Argentina & 13 & 0 & $\begin{array}{c}8 \\
(61,5)\end{array}$ & $\begin{array}{c}9 \\
(69,2)\end{array}$ & $\begin{array}{c}8 \\
(61,5)\end{array}$ & $\begin{array}{c}4 \\
(30,8)\end{array}$ & $\begin{array}{c}3 \\
(23,1)\end{array}$ & $\begin{array}{c}6 \\
(46,2)\end{array}$ \\
\hline Colombia & 12 & 0 & $\begin{array}{c}8 \\
(66,7)\end{array}$ & $\begin{array}{c}9 \\
(75)\end{array}$ & $\begin{array}{c}9 \\
(75)\end{array}$ & $\begin{array}{c}7 \\
(58,3)\end{array}$ & $\begin{array}{c}5 \\
(41,7)\end{array}$ & $\begin{array}{c}2 \\
(16,7)\end{array}$ \\
\hline Perú & 13 & $\begin{array}{c}1 \\
(7,7)\end{array}$ & $\begin{array}{c}2 \\
(15,4)\end{array}$ & $\begin{array}{c}1 \\
(7,7)\end{array}$ & $\begin{array}{c}1 \\
(7,7)\end{array}$ & $\begin{array}{c}2 \\
(15,4)\end{array}$ & $\begin{array}{c}1 \\
(7,7)\end{array}$ & $\begin{array}{c}2 \\
(15,4)\end{array}$ \\
\hline Ecuador & 5 & 0 & $\begin{array}{c}3 \\
(60)\end{array}$ & $\begin{array}{c}2 \\
(40)\end{array}$ & $\begin{array}{c}2 \\
(40)\end{array}$ & $\begin{array}{c}1 \\
(20)\end{array}$ & $\begin{array}{c}1 \\
(20)\end{array}$ & $\begin{array}{c}1 \\
(20,0)\end{array}$ \\
\hline Total & 58 & $\begin{array}{c}1 \\
(1,7)\end{array}$ & $\begin{array}{c}31 \\
(53,4)\end{array}$ & $\begin{array}{c}29 \\
(50)\end{array}$ & $\begin{array}{c}28 \\
(48,3)\end{array}$ & $\begin{array}{c}18 \\
(31)\end{array}$ & $\begin{array}{c}10 \\
(17,2)\end{array}$ & $\begin{array}{c}14 \\
(24,1)\end{array}$ \\
\hline
\end{tabular}

Piezas promocionales de MVL recolectadas en farmacias y la vía pública ${ }^{a}$

\begin{tabular}{|c|c|c|c|c|c|c|c|c|}
\hline Nicaragua & 3 & 0 & $\begin{array}{c}3 \\
(100)\end{array}$ & $\begin{array}{c}2 \\
(66,7)\end{array}$ & $\begin{array}{c}2 \\
(66,7)\end{array}$ & $\begin{array}{c}1 \\
(33,3)\end{array}$ & $\begin{array}{c}1 \\
(33,3)\end{array}$ & $\begin{array}{c}2 \\
(66,7)\end{array}$ \\
\hline Argentina & 8 & $\begin{array}{c}2 \\
(25)\end{array}$ & $\begin{array}{c}8 \\
(100)\end{array}$ & $\begin{array}{c}8 \\
(100)\end{array}$ & $\begin{array}{c}8 \\
(100)\end{array}$ & $\begin{array}{c}5 \\
(62,5)\end{array}$ & $\begin{array}{c}2 \\
(25)\end{array}$ & $\begin{array}{c}6 \\
(75)\end{array}$ \\
\hline Colombia & 9 & $\begin{array}{c}4 \\
(44,4)\end{array}$ & $\begin{array}{c}8 \\
(88,9)\end{array}$ & $\begin{array}{c}6 \\
(66,7)\end{array}$ & $\begin{array}{c}6 \\
(66,7)\end{array}$ & $\begin{array}{c}7 \\
(77,8)\end{array}$ & 0 & $\begin{array}{c}1 \\
(11,1)\end{array}$ \\
\hline Perú & 13 & 0 & $\begin{array}{c}9 \\
(69,2)\end{array}$ & $\begin{array}{c}2 \\
(15,4)\end{array}$ & $\begin{array}{c}5 \\
(38,5)\end{array}$ & $\begin{array}{c}7 \\
(53,8)\end{array}$ & $\begin{array}{c}1 \\
(7,7)\end{array}$ & $\begin{array}{c}7 \\
(53,8)\end{array}$ \\
\hline Ecuador & 1 & 0 & $\begin{array}{c}1 \\
(100)\end{array}$ & 0 & 0 & 0 & 0 & $\begin{array}{c}1 \\
(100,0)\end{array}$ \\
\hline
\end{tabular}

Fuente: elaboración de los autores.

Nota: MVP: medicamentos de venta con prescripción; MVL: medicamentos de venta libre.

a En establecimientos de salud solo se recogieron en total seis piezas promocionales de MVL: 1 en Argentina, 1 en Colombia, 2 en Ecuador y 2 en Perú; en dos casos se omitía información sobre precauciones y advertencias, y en cuatro se enunciaban indicaciones no aprobadas por la autoridad sanitaria correspondiente.

ciones no autorizadas, tanto para MVP como MVL, dado el riesgo potencial para la salud que entraña usar medicamentos con indicaciones con poca o nula evidencia sobre su beneficio (31). La presencia de esta información incumple las regulaciones de los países consultados y los criterios éticos promovidos por la OMS, los cuales destacan que toda información debe ceñirse estrictamente a la ficha técnica aprobada por la autoridad sanitaria correspondiente (1).
Esta investigación demuestra que las estrategias de promoción no distinguen la condición de venta del medicamento (MVL o MVP), situación que debería preocupar a las autoridades y que constituye una alerta a las capacidades de vigilancia y monitoreo de los organismos oficiales responsables. Asimismo, es un llamado de atención sobre la importancia de revisar y analizar las tendencias de reclasificación de MVL/ MVP para ampliar la difusión promo- cional en medios de comunicación masiva.

También se debe señalar el riesgo de la promoción como inductora de consumo, dado que en los anuncios publicitarios las indicaciones del medicamento son la información más frecuentemente incluida, en claro contraste con la ausencia de información relacionada con su seguridad. Esta circunstancia podría limitar la evaluación del riesgo-beneficio global del producto al momento de tomar una 
CUADRO 5. Riesgo relativo (RR) de la información contenida en las piezas promocionales de medicamentos seleccionadas y analizadas en cinco países de América Latina

\begin{tabular}{lll}
\hline Variable & RR & IC95\% \\
\hline
\end{tabular}

Piezas de MVP halladas en farmacias versus establecimientos de salud

$\begin{array}{lcc}\text { Indicaciones } & 10,24 & 1,29-81,46 \\ \text { Reacciones adversas a medicamentos } & 1,43 & 1,06-1,94 \\ \text { Precauciones y advertencias } & 1,29 & 0,91-1,85 \\ \text { Contraindicaciones } & 1,34 & 0,93-1,93 \\ \text { Posología } & 2,08 & 1,32-3,29 \\ \text { Forma farmacéutica } & 1,02 & 0,41-2,57 \\ \text { Indicaciones no aprobadas }^{a} & 1,1 & 0,53-2,26\end{array}$

Piezas (halladas en farmacias) de MVP versus MVL

\begin{tabular}{|c|c|c|}
\hline Indicaciones & 1,0 & $0,36-2,79$ \\
\hline Reacciones adversas a medicamentos & 0,90 & $0,71-1,13$ \\
\hline Precauciones y advertencias & 1,22 & $0,82-1,83$ \\
\hline Contraindicaciones & 1,05 & $0,73-1,51$ \\
\hline Posología & 1,10 & $0,76-1,60$ \\
\hline Forma farmacéutica & 1,50 & $0,46-4,84$ \\
\hline Indicaciones no aprobadas ${ }^{a}$ & 0,53 & $0,28-1,02$ \\
\hline
\end{tabular}

Fuente: elaboración de los autores.

Nota: IC95\%: intervalo de confianza de 95\%; MVP: medicamentos de venta con prescripción; MVL: medicamentos de venta libre.

a Por la autoridad sanitaria correspondiente.

decisión terapéutica, tanto por parte de quien prescribe -en el caso de los MVP - como del consumidor, si se trata de MVL.

Se encontró que en ninguno de los cinco países estudiados se observa la regulación que restringe la promoción de MVP (23-27, 32). Tal incumplimiento se esconde detrás de métodos publicitarios disimulados, como la exposición de afiches en sitios sanitarios donde concurre la población general (p. ej. hospitales y farmacias) o la distribución de "información para educación" directamente a los pacientes. Como ya se ha demostrado en otros estudios, esta práctica resulta efectiva como mecanismo de persuasión para el consumo $(33,34)$.

Argentina, país que cuenta con un marco regulatorio de promoción farmacológica coherente, es el que mostró la tasa más baja de cumplimiento de las regulaciones, en particular con respecto a la mención -en anuncios publicitarios- de indicaciones no aprobadas por el organismo de salud pertinente. Tal hallazgo confirma la función crucial que cumple la vigilancia activa de la P y P de fármacos, así como la necesidad de fortalecer las actividades de control y monitoreo, lo que podría conseguirse por ejemplo mediante redes de apoyo de universidades, organizaciones no gubernamentales y otras entidades que trabajan en este campo.
El presente estudio constituye un primer acercamiento a la situación de la publicidad farmacéutica en América Latina, representada aquí por cinco de sus países. En vista de sus resultados, hará falta realizar nuevos trabajos enfocados a temas específicos tales como el papel crítico de la sociedad civil en el control de la promoción de medicamentos y el seguimiento de la aplicación de las sanciones establecidas en cada país. Será necesario hacer hincapié en el impacto que tienen las regulaciones en el consumo de medicamentos y la salud de la población, en los procedimientos de aprobación —revisión previa o posterior a su publicación - de la promoción farmacéutica, en los efectos de la tendencia internacional a permitir la publicidad directa al consumidor y en la adopción de códigos de autorregulación por parte de la industria (35-37).

El análisis de la literatura permitió identificar otros elementos deseables para incluir en las regulaciones, entre ellos la rectificación pública cuando se comprueba que la información divulgada es engañosa o ambigua y el precio de los medicamentos en el material promocional $(27,38)$. Adicionalmente, las regulaciones deberían abordar datos relevantes sobre los ensayos clínicos citados en la bibliografía de los materiales para el personal médico, tales como significación estadística y validez clínica de los resultados, número mínimo de las muestras de pacientes participantes en los ensayos, número necesario de pacientes para causar un efecto adverso (NNH por sus siglas en inglés) y riesgo absoluto, así como prohibir el uso de cartas de recomendación o agradecimiento dirigidas a terceros, para contrarrestar el efecto de los líderes de opinión en las prácticas de prescripción (9).

\section{Limitaciones del estudio}

Dado que una regulación puede perder actualidad fácilmente, se decidió analizarla en función de sus brechas con los criterios éticos de la OMS, por ser contenidos técnicos internacionales con mayores probabilidades de vigencia a lo largo del tiempo. Vale señalar asimismo que los hallazgos, aun cuando están basados en un análisis adecuado de los datos obtenidos, se limitan a los cinco países estudiados y, por tanto, no pueden generalizarse a la situación de toda América Latina. Por otra parte, pese a que la muestra se recolectó en un lapso relativamente corto (un mes), su tamaño (683 piezas) y la posterior selección al azar de las 132 piezas analizadas permiten considerarla representativa de los materiales publicitarios impresos por lo común disponibles tanto para los profesionales de la salud como para la población en general.

\section{Conclusiones}

Aun cuando en general los cinco países del estudio incorporan en sus regulaciones sobre P y P de medicamentos las recomendaciones de la OMS, con frecuencia dichas ordenanzas no se reflejan en los contenidos de las piezas promocionales. Adicionalmente, en la muestra del estudio se observó que no hay diferencia en los contenidos de las piezas promocionales según la condición de venta del producto (MVP y MVL) y que incluyen abundante información sobre las indicaciones del medicamento -en contraste con la escasa o inexistente información relacionada con su seguridad. El magro cumplimiento de las regulaciones emitidas por las autoridades de salud en materia de promoción farmacéutica pone en evidencia la necesidad que tienen los países de revisar y fortalecer tanto las especificaciones de dichas ordenanzas como los procedimientos para fiscalizar su acatamiento y aplicar las sanciones correspondientes. 
Agradecimientos. Los autores quieren agradecer la contribución y el respaldo de los siguientes miembros del Grupo de Análisis y Vigilancia de la Promoción
Farmacéutica de Acción Internacional para la Salud: Germán Rojas (Perú), José Augusto Cabral de Sobravime (Brasil), José Terán (Ecuador), Benoit Marchand y Carlos Fuentes (Nicaragua), Óscar Lanza y René Soria (Bolivia), Cecilia Homar (Argentina) y Francisco Rossi (Colombia).

\section{REFERENCIAS}

1. Organización Mundial de la Salud. Criterios éticos para la promoción de medicamentos (Resolución WHA 41.17). Ginebra: OMS; 1988.

2. Organización Mundial de la Salud. Criterios éticos de la OMS para la promoción de medicamentos (Resolución WHA 45.30). Ginebra: OMS; 1992.

3. Organización Mundial de la Salud. Criterios éticos de la OMS para la promoción de medicamentos (Resolución WHA 47.16). Ginebra: OMS; 1994.

4. Organización Mundial de la Salud. Promoción, publicidad y venta transfronterizas de productos médicos por internet (Resolución WHA 51.9). Ginebra: OMS; 1998.

5. Chilet-Rosell E, Llaguno MM, Cantero MT, Alonso-Coello P. Hormone replacement therapy advertising: sense and nonsense on the web pages of the best-selling pharmaceuticals in Spain. BMC Public Health. 2010;10:134.

6. Organización Mundial de la Salud. Uso racional de los medicamentos: progresos realizados en la aplicación de la estrategia farmacéutica de la OMS. Reunión 118 del Consejo Ejecutivo, Informe de la Secretaría (Reporte No.: EB118/6; 2006).

7. Consumers International. La salud patentada. La perspectiva del consumidor sobre la RSE, la promoción de medicamentos y la industria farmacéutica en Europa. Londres: Consumers International; 2006. Hallado en: http:/ / www. consumersinternational.org/media/286105/ salud_patentada.pdf. Acceso el 24 de diciembre de 2010.

8. Satue E. Publicidad directa al consumidor en la UE. Essential drugs; 2002. Hallado en: http://www.essentialdrugs.org/emed/archi ve/200206/msg00000.php. Acceso el 24 de diciembre de 2010.

9. Ministério da Saúde, Brasil. Estudo comparado regulamentação da propaganda de medicamentos. 1. ${ }^{a}$ Edição. Basilia D.F.: Ministério da saúde; 2005. Hallado en: http:// www.ccs.saude.gov.br/visa/publicacoes/ arquivos/Estudo_comparado_propaganda. pdf. Acceso el 24 de diciembre de 2010.

10. Asociación de Laboratorios Farmacéuticos de Investigación (AFIDRO), Colombia. Código de ética. Colombia: Buenos \& Creativos E.U.; 2007.

11. Heinrich J. US Prescription drugs. FDA Oversight to direct-to-consumer advertising has limitations. Report to Congressional Requesters. US General Accounting Office. GAO-03-177; 2002. Hallado en: http:/ / www. gao.gov/new.items/d03177.pdf. Acceso el 24 de diciembre de 2010

12. Woloshin S, Schwartz LM, Tremmel J, Welch HG. Direct-to-consumer advertisements for prescription drugs: what are Americans being sold. Lancet. 2001;358:1141-6.

13. Cassels A, Hughes MA, Cole C, Mintzes B, Lexchin J, McCormack JP. Drugs in the news: an analysis of Canadian newspaper coverage of new prescription drugs. CMAJ. 2003;168: $1133-7$.

14. Toop L, Richards D. New Zealand deserves better. Direct-to-consumer advertising (DTCA) of prescription medicines in New Zealand: for health or for profit? NZMJ. 2003;116:1-6.

15. Caffaratti M, Briñón M. Atención con la publicidad de medicamentos. Centro de Información de Medicamentos, Departamento de Farmacia, Facultad de Ciencias Químicas, Universidad Nacional de Córdoba (UNC). Boletín no. 17. Córdoba, Argentina: UNC; 2003.

16. Domosbian D. Seminario del Instituto de Investigaciones Farmacológicas. Publicidad de medicamentos en los medios: una cuestión preocupante. Hallado en: http://www.essen tialdrugs.org/efarmacos/archive/200405/m sg00037.php. Acceso el 24 de diciembre de 2010.

17. Kravitz RL, Epstein RM, Feldman MD, Franz $\mathrm{CE}$, Azari R, Wilkes MS, et al. Influence of patients requests for direct-to-consumer advertised antidepressants a randomized controlled trial. JAMA. 2005;293:1995-2002.

18. Acción Internacional para la Salud Latinoamérica \& Caribe (AIS). Conflicto de intereses: un asunto siempre presente. Traducido de: Worst Pills: "Conflicts of interest: an issue that will not go away"; 2007. Hallado en: http://aislac.org/index.php?option= com content\&view=article\&id=346: conflictode-intereses-un-asunto-siempre-presente\& catid=26:noticias $-2007 \&$ Itemid $=154$. Acceso el 24 de diciembre de 2010.

19. Alonso V. Consumo de medicamentos y equidad en materia de salud en el Área Metropolitana de Buenos Aires, Argentina. Rev Panam Salud Publica. 2003;13(6):400-6.

20. British Medical Association. British National Formulary. 54th ed. London: BMA; 2007.

21. Sweetman SC. Martindale: the complete drug reference. Electronic version. London: Pharmaceutical Press; 2007.

22. Food and Drug Administration (FDA), Center for Drug Evaluation and Research, US. Drugs at FDA: FDA Approved drug products. Hallado en: http://www.accessdata. fda.gov/scripts/cder/drugsatfda/index.cfm. Acceso el 24 de diciembre de 2010.

23. Administración Nacional de Medicamentos (ANMAT), Argentina. Normas generales y específicas que deberá cumplir toda publicidad o propaganda dirigida al público, cuyo objeto sea promocionar especialidades medicinales de venta libre, productos alimenticios, cosméticos, para la higiene personal y perfume, domisanitarios, odontológicos, para diagnósticos de uso in vitro, suplementos dietarios y dispositivos de tecnología médica. Disposición no. 4980/2005. Argentina: ANMAT; 2005.
24. Ministerio de la Protección Social, Colombia. Reglamentación de la publicidad de medicamentos y productos fitoterapéuticos de venta sin prescripción facultativa o de venta libre. Resolución no. 4320 de 2004.

25. Comisión Legislativa Nacional, Ecuador. Ley Orgánica de Salud (ley no. 67, 2006).

26. Asamblea Nacional, Nicaragua. Ley de Medicamentos y Farmacias (ley no. 292, 1998).

27. Congreso de la República, Perú. Ley General de Salud (ley no. 26842, 1997).

28. Vacca C, Vargas C, Cañás M. Regulación de la publicidad y promoción farmacéutica (P y P) en cinco países de América Latina. Presentado en el VI Encuentro Internacional de Farmacovigilancia. Bogotá, Colombia; 2009.

29. Zapata D, Vacca C. Análisis comparativo sobre la regulación de la publicidad y promoción de medicamentos en Colombia y otros países de América Latina. Trabajo de grado, Departamento de Farmacia, Universidad Nacional de Colombia; 2007.

30. Mintzes B. Disease mongering in drug promotion: do governments have a regulatory role? PLoS Med. 2006;3:e198.

31. Martín-Llaguno M, Álvarez-Dardet C. Withdrawal of an advertising campaign to promote the quadrivalent human papilloma virus vaccine in Spain. Gac Sanit. 2010;24: 75-7.

32. Ministerio de Salud Pública, Argentina. Buenas prácticas de promoción de medicamentos de venta bajo receta (resolución no. 627, modificación de la resolución no. 1061, 2007).

33. Food and Drug Administration (FDA). FDA Warns individuals and firms to stop selling fake cancer 'cures' fraudulent claims on internet sites. Hallado en: http://test.fda.gov/ NewsEvents/Newsroom/PressAnnounce ments/2008/ucm1046087.htm. Acceso el 24 de diciembre de 2010.

34. Medawar C. Direct to consumer advertising: a cynical consultation exercise? BMJ. 2008;336 (7648):787.

35. Shapiro MF. Regulating pharmaceutical advertising: what will work? CMAJ. 1997;156: 359-61.

36. House of Commons Health Committee. The influence of the pharmaceutical industry. Fourth report of session 2004-05, vol. 1. London: The Stationery Office; 2005.

37. Thawani V. Drug promotion: can self-regulation work? Indian J Pharmacol. 2002;34:227-8.

38. Ministerio de Salud y Deportes, Bolivia. Normas éticas para la promoción de medicamentos. Bolivia: Ministerio de Salud y Deportes; 2000.

Manuscrito recibido el 14 de mayo de 2010. Aceptado para publicación, tras revisión, el 1 de noviembre de 2010. 
ABSTRACT Objective. To analyze differing regulations regarding drug promotion, and the extent of compliance as seen in samples of advertising directed to the public in Argentina, Colombia, Ecuador, Nicaragua, and Peru.

Drug advertising and Methods. A total of 683 pieces of promotional material on display in health facilities, pharmacies, and on the street were collected, 132 of which were randomly selected for analysis. The regulations governing pharmaceutical advertising, taken from official websites and interviews with regulatory officials and Ministry of Health staff in the five countries covered, were reviewed, along with their adherence to the ethical criteria of the World Health Organization (WHO). The contents of the materials in the sample were evaluated to determine their degree of compliance with national regulations and WHO recommendations on drug promotion.

Results. The countries have regulations incorporating $\mathrm{WHO}$ ethical criteria. Over $80 \%$ of the material analyzed included the indications for the drug, while over $70 \%$ omitted information on adverse effects. Fifty percent of the advertisements for overthe-counter (OTC) drugs on display in pharmacies listed indications not approved by the relevant health authority. In advertising in pharmacies, the risks from inadequate information were not found to differ significantly for OTC or prescription medications. Compared with materials provided in health facilities, the relative risk of the absence of information on dosage in the material distributed in pharmacies was 2.08 (confidence interval 95\% 1.32-3.39).

Conclusions. Although regulations on drug promotion and advertising in the five countries studied generally incorporate the WHO recommendations, promotional materials often fail to reflect the fact.

Key words Drug publicity; ethics, pharmacy; products publicity control; nonprescription drugs; prescription drugs; Argentina; Colombia; Ecuador; Nicaragua; Peru; Latin America. 